\title{
Direct Visualization and Characterization of Interfacially Adsorbed Polymer atop Nanoparticles and within Nanocomposites
}

\author{
Katelyn Randazzo, Malgorzata Bartkiewicz, Bartlomiej Graczykowski, Daniele Cangialosi, George Fytas, \\ Biao Zuo,* and Rodney D. Priestley*
}

Cite This: Macromolecules 2021, 54, 10224-10234

Read Online

ABSTRACT: Irreversible adsorption at polymer/substrate interfaces has been reported to influence glassy properties in thin films. However, consideration has yet to be extended to the nanocomposite geometry, wherein a large interfacial area and high processing temperatures afford especially favorable conditions for irreversible adsorption at the polymer/nanoparticle interface. Here, we present an approach for directly measuring the site-specific glassy properties at the polystyrene (PS)-adsorbed layer interface in PS-silica nanocomposites. We achieved this using a stepwise assembly approach to localize fluorescent dyes within the nanocomposite adsorbed layer, subsequently measuring the glass transition temperature $\left(T_{\mathrm{g}}\right)$ via fluorescence. We found that PS adsorption within nanocomposites strongly influenced the local $T_{\mathrm{g}}$. By measuring the thickness of the PSadsorbed layers atop nanoparticles via transmission electron microscopy, we found a correlation between adsorbed layer $T_{\mathrm{g}}$ and thickness. Our results provide compelling evidence that adsorbed layer formation within polymer nanocomposites can have a profound impact on local interfacial properties.

\section{INTRODUCTION}

Polymer nanocomposites feature an array of propertiesincluding enhanced mechanical strength, ${ }^{1-3}$ thermal stability, $^{3,4}$ and ductility ${ }^{2}$ - which are superior to those of analogous unfilled polymers. The origin of these enhanced physical properties is generally considered to be perturbed local properties at the polymer/filler interface, which-due to the large specific surface area, even for small amounts of nanofiller-can potentially exert a strong governing influence on the bulk properties of the overall nanocomposite. A prominent example of local perturbation at the polymer/ nanofiller interface is that arising from the formation of an adsorbed layer, ${ }^{5}$ a nanoscale region wherein favorable interfacial interactions, typically facilitated by annealing above the glass transition temperature $\left(T_{\mathrm{g}}\right)$, enable polymer chains to overcome the entropic penalties associated with restricted chain conformational freedom, and ultimately physically adhere atop an adjacent surface. ${ }^{6-10}$ Several conceptions of the irreversibly adsorbed layer have been proposed-from a completely immobilized or "dead" layer which essentially does not participate in the glass transition ${ }^{11,12}$ to a region of dramatically slowed but nevertheless mobile chains to a region wherein packing frustration promotes excess free volume and accordingly a reduction in $T_{\mathrm{g}}$ compared to that of the bulk polymer. ${ }^{9,13,14}$ While these conceptions may vary in their suitability for describing various polymer/ substrate pairings, they are generally similar in their treatment of the irreversibly adsorbed or "bound" layer as a constant feature of the system.

Adsorbed layer formation is strongly dependent upon processing conditions. Due to governing chain kinetics during a typical experimental timescale, adsorption occurs more readily at elevated temperatures, ${ }^{8}$ coinciding for practical purposes with standard processing parameters. ${ }^{15-17}$ Moreover, longer annealing times elicit adsorbed layers of greater thickness. ${ }^{6,9,18}$ The processing-dependent nature of irreversibly adsorbed layers has important implications in polymer nanocomposites, whose preparation typically involves a sustained high-temperature annealing step. ${ }^{19-24}$ Taken together with the large specific surface area of polymer nanocomposites, the temperature dependence of adsorption suggests that adsorbed layers may significantly contribute to modifying nanocomposite properties. However, direct investigations of irreversible adsorption-its evolution and its consequences-have not yet extended beyond polymer thin films $s^{9,25}$ to consider polymer nanocomposites. Definitive inferences about adsorption in nanocomposites are not readily made from extant reports of nanocomposite properties. The

Received: July 23, 2021

Revised: September 29, 2021

Published: October 28, 2021 
literature, while substantial, encompasses a broad range of varied fabrication procedures, obscuring key details of adsorbed layer formation. Such variations may account for disparate reports of $T_{\mathrm{g}}$ perturbations in seemingly similar systems. For example, the introduction of silica nanoparticles has been observed to cause polystyrene (PS) $T_{\mathrm{g}}$ to increase, ${ }^{26}$ decrease, ${ }^{27,28}$ and remain essentially unchanged. ${ }^{20,29,30}$ Analogous discrepancies have been reported for other pairings, such as silica/poly(methyl methacrylate) (PMMA) (increased, ${ }^{30,31}$ decreased, ${ }^{28,30,32}$ and essentially unchanged ${ }^{31} T_{\mathrm{g}}$ ) and alumina/poly(2-vinylpyridine) (strongly ${ }^{30}$ or very weakly ${ }^{33}$ increased $T_{\mathrm{g}}$ ).

While irreversibly adsorbed layers in polymer nanocomposites warrant thorough consideration, their characterization is not straightforward. Differential scanning calorimetry (DSC) of nanocomposites can inform our understanding of bulk properties but yields information averaged across an entire system. More sophisticated techniques such as modulated DSC, dielectric spectroscopy, and neutron scattering enable somewhat more precise measurements and have supplied evidence of regions of locally perturbed properties in polymer nanocomposites. ${ }^{34-38}$ However, such measurements remain indirect, leaving parameters such as layer thickness to be inferred based on estimates of local density. One of the more useful strategies for elucidating local polymer properties inaccessible by conventional techniques is fluorescence spectroscopy, ${ }^{9,20,39-41}$ wherein fluorescent labels at specific locations within a polymeric material can directly convey information about local properties such as $T_{\mathrm{g}}$ at nanoscale resolution. This approach has been applied to study polymer thin films ${ }^{42-45}$ and multilayer films composed of fluorescently labeled and unlabeled components, ${ }^{43,46,47}$ facilitating valuable insights into local $T_{\mathrm{g}}$ gradients under confinement. Subsequent work has extended the applicability of fluorescence spectroscopy toward more complex systems, with investigations encompassing bulk aging behavior of polymer nanocomposites, ${ }^{30,38}$ spatial gradients of $T_{\mathrm{g}}$ in "model" polymer nanocomposites consisting of polymer films encased between two flat supporting surfaces, ${ }^{12,40}$ and interfacial $T_{\mathrm{g}}$ of nanoparticles as measured by surface-grafted fluorescent labels. $^{20}$

Given the range of techniques available, the key challenges in characterizing irreversible adsorption in polymer nanocomposites are developing a means of extracting thicknessrelated information from the adsorbed layers and conducting an in situ investigation of glassy properties in this nanoscale region alone. Here, we report an approach that leverages transmission electron microscopy (TEM) and fluorescence spectroscopy to directly investigate, respectively, growth of the adsorbed layer at the nanoparticle interface and the corresponding changes in local glass transition behavior in nanocomposites. As we will show in this work, by supplementing these techniques with a stepwise assembly approach to localize fluorescent dyes, we are able to directly access the features and dynamics of irreversibly adsorbed layers within nanocomposites, thus ultimately overcoming the most limiting obstacles to detailed characterization of local adsorption in such a system. Additionally, adjusting the processing conditions allowed us to directly characterize the influence of irreversible adsorption on local $T_{\mathrm{g}}$ within a polymer nanocomposite, an important but often-overlooked aspect of nanocomposite preparation and manufacture. We found that chain adsorption on the surfaces of particles greatly affected local $T_{\mathrm{g}}$ of the polymers and that the correlation between the adsorbed layer size and local $T_{\mathrm{g}}$ was reliably described by the free volume holes diffusion (FVHD) model. We present this approach for direct characterization of the adsorbed layer in nanocomposites with the intention that it enables new fundamental insights into irreversible adsorption and serves as a platform for developing valuable material properties.

\section{EXPERIMENTAL SECTION}

Synthesis and Characterization of Polystyrene and Fluorescently Labeled Polystyrene. PS $\left(M_{\mathrm{w}}=218 \mathrm{~kg} / \mathrm{mol}\right.$ and $M_{\mathrm{w}} / M_{\mathrm{n}}$ $=1.67)$ and fluorescently labeled PS $\left(M_{\mathrm{w}}=230 \mathrm{~kg} / \mathrm{mol}\right.$ and $M_{\mathrm{w}} / M_{\mathrm{n}}=$ 1.69) were synthesized by benzoyl peroxide-initiated bulk free radical polymerization of styrene (Sigma-Aldrich) at $80{ }^{\circ} \mathrm{C}$. The starting materials for fluorescently labeled PS included a small amount $(<0.1$ mol \%) of the fluorescent monomer 1-pyrenylbutyl methacrylate, which had been synthesized via the esterification of methacryloyl chloride (Sigma-Aldrich) and 1-pyrenebutanol (Aldrich) in a solution of triethylamine (Sigma-Aldrich) and dry tetrahydrofuran (THF) (Sigma-Aldrich) and then purified by recrystallization in ethanol (Fisher Scientific). ${ }^{43}$ The as-synthesized polymers were purified three times each by dissolution in toluene (Fisher Chemical) and precipitation in methanol (Fisher Chemical) to remove the residual monomer. The molecular weights and dispersities of the polymers were characterized relative to PS standards using size exclusion chromatography. Bulk $T_{\mathrm{g}}$ was measured on second heating at $2{ }^{\circ} \mathrm{C} /$ min via DSC on a TA Instruments Q2000 DSC. Bulk $T_{\mathrm{g}}$ values of neat PS $\left(104.0{ }^{\circ} \mathrm{C}\right)$ and neat labeled PS $\left(106.3{ }^{\circ} \mathrm{C}\right)$ were consistent with each other and with the bulk $T_{\mathrm{g}}$ value acquired by fluorescence spectroscopy $\left(106.0^{\circ} \mathrm{C}\right)$.

Synthesis of Silica Nanoparticles. Silica nanoparticles $\left(\mathrm{SiO}_{x}\right.$ $\mathrm{NPs}$ ) were prepared according to the one-step Stöber process ${ }^{48}$ using tetraethyl orthosilicate (Aldrich), ethanol (Fisher Scientific), ammonium hydroxide (Fisher Chemical), and deionized water. Scanning electron microscopy (SEM) and TEM imaging of the asprepared $\mathrm{SiO}_{x} \mathrm{NPs}$ revealed them to be spherical and monodisperse (see Figure S1), with an average diameter $d_{\mathrm{NP}}$ of $289 \pm 12 \mathrm{~nm}$ (corresponding to $\sigma / d_{\mathrm{NP}}=0.0018$ ), based on measurements of 84 TEM-imaged nanoparticles in three directions each. Prior to preparation of the polymer nanocomposites, the water in the $\mathrm{SiO}_{x}$ suspension was exchanged for dimethylformamide (DMF), which is a good solvent for PS and also charge-stabilizes the $\mathrm{SiO}_{x} \mathrm{NPs}$ to prevent the formation of aggregates. ${ }^{19}$

Preparation of Polymer Nanocomposites with Local Fluorescence. A polymer nanocomposite consisting of $\mathrm{PS}, \mathrm{SiO}_{x}$ NPs, and fluorescently labeled PS localized specifically in irreversibly adsorbed regions was prepared via a stepwise assembly. An initial polymer nanocomposite with $4 \mathrm{wt} \%$ silica loading was prepared from solution of labeled PS and a DMF suspension of $289 \mathrm{~nm} \mathrm{SiO}{ }_{x} \mathrm{NPs}$. Preparation generally followed the procedure outlined by Meth and co-workers involving casting of a viscous solution of $\mathrm{SiO}_{x} \mathrm{NPs}, \mathrm{DMF}$, and high- $M_{\mathrm{w}}$ PS, wherein inhibited diffusion of nanoparticles served to enhance dispersion. ${ }^{19}$ After annealing the polymer at $156{ }^{\circ} \mathrm{C}\left(\sim T_{\mathrm{g}}\right.$ $+50 \mathrm{~K})$ for a specified adsorption time, the nanoparticles bearing adsorbed layers were separated from the unadsorbed matrix and washed thoroughly. (This procedure is summarized in Figure S2.) The polymer nanocomposite was initially dissolved in toluene, sonicated for $30 \mathrm{~min}$, and centrifuged at $13,000 \mathrm{rpm}$ for $5 \mathrm{~min}$. After subsequent removal of the supernatant containing the unadsorbed free polymer, there remained a pelletized mass of $\mathrm{SiO}_{x}$ NPs coated in the irreversibly adsorbed polymer. These nanoparticles were resuspended in fresh toluene using a vortex mixer and, once again, subjected to sonication for $30 \mathrm{~min}$, centrifugation at 13,000 $\mathrm{rpm}$ for $5 \mathrm{~min}$, removal of the supernatant, and resuspension in fresh toluene. In total, four washing cycles of sonication and centrifugation were found to be sufficient to completely remove the unadsorbed polymer from the nanoparticles (see Figure $1 \mathrm{~b}$ and related discussion below). However, for thoroughness, the nanoparticles underwent five 
(a)

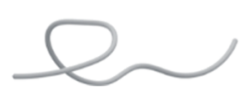

Polystyrene

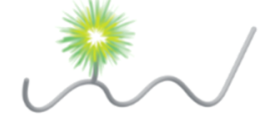

Fluorescently-labeled polystyrene

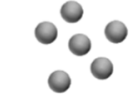

Monodisperse silica nanoparticles
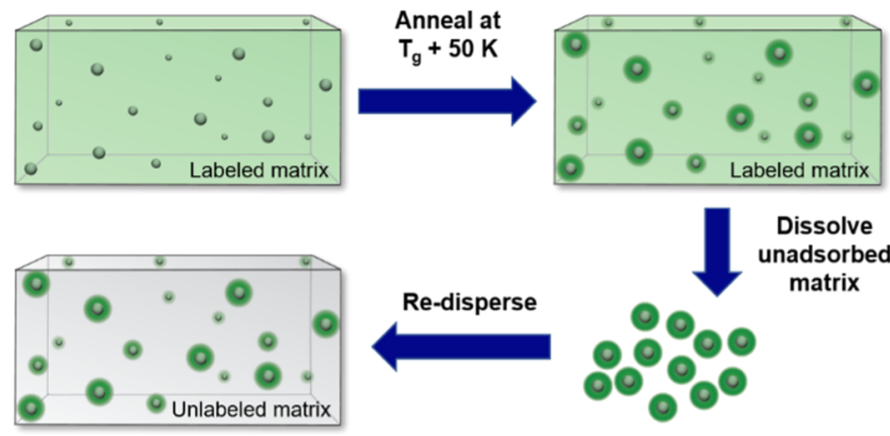

(b)

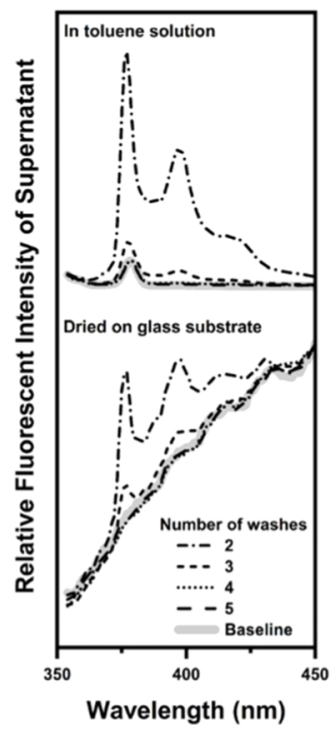

(c)

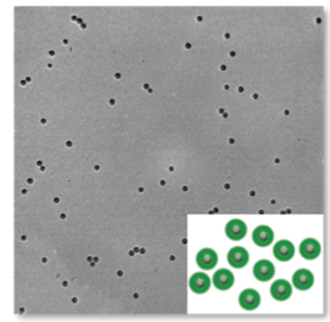

(d)

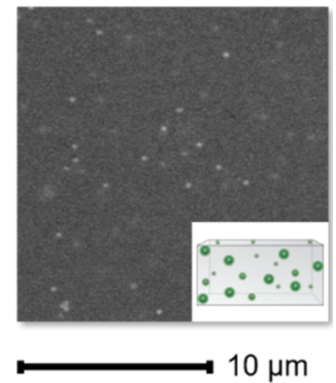

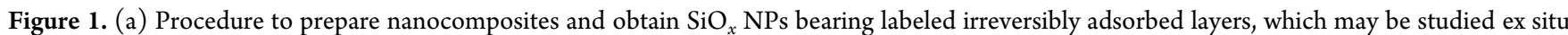
or redispersed within an unlabeled polymer matrix to produce polymer nanocomposites with fluorescent dyes isolated within the irreversibly adsorbed layers. (b) Fluorescence emission of the supernatant, both in solution (top) and dried (bottom) forms, following dissolution of the unadsorbed matrix. Spectra indicate that the unadsorbed polymer was completely removed after four washing cycles. Baseline spectra refer to pure toluene and a clean glass substrate. For both sets of spectra, the emission spectrum of the supernatant after the first washing cycle is at least 2 orders of magnitude higher than those of the subsequent washing cycles and thus for clarity is not shown here. (c) TEM image showing washed $\mathrm{SiO}_{x} \mathrm{NPs}$ bearing adsorbed layers. (d) SEM image of $\mathrm{SiO}_{x} \mathrm{NPs}$ bearing adsorbed layers and redispersed in a new matrix. Although the adsorbed layers and matrix are composed of pyrene-labeled and unlabeled PS, respectively, the NPs exhibit good dispersion in the resulting nanocomposite.

washing cycles. The washed nanoparticle solutions were then either drop-cast onto TEM grids or glass substrates or combined with unlabeled PS and cast onto glass substrates to yield 1 wt $\%$ silica/PS nanocomposites wherein nanoparticles bearing irreversibly adsorbed layers of labeled PS were dispersed in a matrix of unlabeled PS (see the schematic depicted in Figure 1a). The converse case-wherein the adsorbed layers and unadsorbed matrix were composed of unlabeled and labeled PS, respectively-was studied in nanocomposites which were prepared according to the analogous, but complementary, procedure (see the schematic depicted in Figure 7). All samples were dried in a vacuum oven.

TEM Characterization of Irreversibly Adsorbed Layers. Suspensions of $\mathrm{SiO}_{x} \mathrm{NPs}$ bearing irreversibly adsorbed layers annealed for different lengths of time were drop-cast on TEM grids and immediately dried under vacuum. The particles were imaged using a Thermo Fisher Scientific Talos F200X TEM instrument. In order to preserve the stability of the adsorbed layers under the TEM beam, samples were loaded into the column using a liquid nitrogenfilled cryo-holder, which was allowed to equilibrate at a low temperature before imaging was conducted under the low-dose mode. Reported values of measured adsorbed layer thickness represent, for each $t_{\mathrm{ann}}$, the average of measurements taken from at least 10 nanoparticles measured in at least two places each.

BLS Characterization of Irreversibly Adsorbed Layers. Brillouin light scattering (BLS) measures the spheroidal $s(1,2)$ vibration mode with frequency $f(1,2)=A c_{t} / d$ for the case of individual spherical nanoparticles with diameter $d$ and transverse sound velocity, $c_{\mathrm{t}}$. $A$ is a constant ( $\sim 0.84$ for a Poisson's ratio of about 0.3 ). The NP shear modulus can be computed from $G=\rho c_{\mathrm{t}}^{2}$, where $\rho$ denotes the NP density. For the case of an individual NP, the displacement field of the single $s(1,2)$ mode resembles an atomic dorbital. For the case of NPs assembled in a close-packed structure, mode displacement field is hybridized due to interparticle interactions, resulting in the split of the fundamental $s(1,2)$ mode into two spectral contributions at $f_{1}$ and $f_{1}<f_{2}$. Furthermore, a new interaction-induced mode $(1,1)$ appears at a lower frequency $f(1,1)$ which is governed by the strength of interactions, the nanoparticle compression modulus, and the contact area. ${ }^{49}$ Simulations have shown that the quantity $f_{\mathrm{L}}=2 f_{1}-f_{2}$ accounts for the presence of interactions, which is well-representative of $f(1,2)$ of noninteracting individual NPs. ${ }^{49}$ The BLS spectra were recorded using a tandemFabry-Pérot interferometer under excitation by $532 \mathrm{~nm}$ laser light. The localized nature of the elastic vibrations is independent of the scattering wavevector, and hence, the spectra were recorded at backscattering geometry.

$T_{\mathrm{g}}$ Characterization by Fluorescence and DSC. Reported $T_{\mathrm{g}}$ values as measured by fluorescence are those of isolated nanoparticles or bulk nanocomposites which were drop-cast from toluene solution onto piranha-treated (soaked in a 70:30 solution of $\mathrm{H}_{2} \mathrm{SO}_{4}$ and $\mathrm{H}_{2} \mathrm{O}_{2}$, at $75^{\circ} \mathrm{C}$ for $1.5 \mathrm{~h}$ ) flat glass substrates and immediately placed into a vacuum oven to dry for $24 \mathrm{~h}$ (isolated nanoparticles) or $72 \mathrm{~h}$ (nanocomposites). The samples were excited at $\lambda=340 \mathrm{~nm}(2.5 \mathrm{~nm}$ slit width), and their steady-state fluorescence emission was collected using a Fluorolog-3 spectrofluorimeter (Horiba Scientific) at $5{ }^{\circ} \mathrm{C}$ intervals upon cooling. Prior to the collection of each spectrum, the samples were temperature-stabilized for $1.5 \mathrm{~min}$, giving an effective cooling rate of $2{ }^{\circ} \mathrm{C} / \mathrm{min}$. $T_{\mathrm{g}}$ was defined to be the intersection of extrapolated linear fits to the temperature-dependent integrated fluorescence emission spectra in the glassy and rubbery regimes. Comparable bulk measurements were obtained via DSC on a TA Instruments Q2000 DSC on second heating following cooling at 2 ${ }^{\circ} \mathrm{C} / \mathrm{min}$.

\section{RESULTS AND DISCUSSION}

Stepwise Nanocomposite Assembly to Obtain Nanoparticles and Nanocomposites with Local Fluorescence. In order to probe the properties unique to the irreversibly adsorbed layer, we employed the scheme shown in Figure 1a to assemble nanocomposites in stepwise stages, that is, by successively preparing the adsorbed and unadsorbed regions. The nanocomposite fabrication process begins with the dispersion of silica nanoparticles $\left(\mathrm{SiO}_{x} \mathrm{NPs}\right)$ into a pyrenelabeled PS matrix followed by high-temperature annealing $\left(\sim T_{\mathrm{g}}+50 \mathrm{~K}\right)$ for a select time to induce the formation of adsorbed nanolayers at the $\mathrm{NP} /$ matrix interface. Subsequent dissolving of the nanocomposite in toluene-a good solvent 

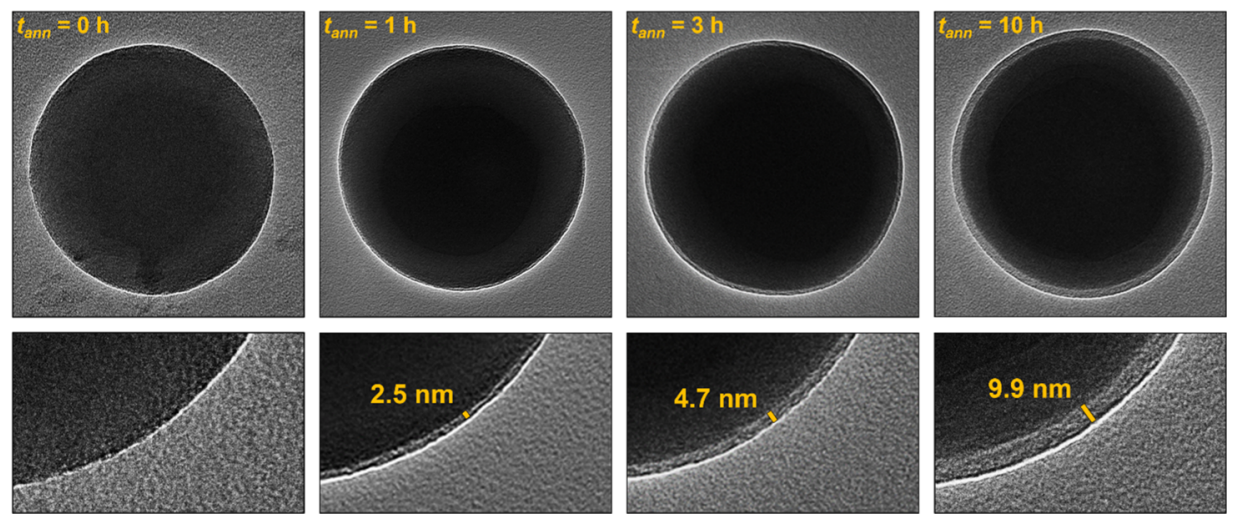

Figure 2. Representative TEM images of $\mathrm{SiO}_{x}$ NPs bearing pyrene-labeled PS-adsorbed layers at different stages of growth. (The faintly visible "core" at the center of each NP is an artifact of TEM grid deformation and does not change as a function of annealing time.)

for PS which does not interfere with $\mathrm{PS} / \mathrm{SiO}_{x}$ interactions to induce desorption ${ }^{18,50}$ - frees NPs of unadsorbed matrix chains but leaves irreversibly adsorbed layers intact. This approach is corroborated by several reports that sustained washing of adsorbed thin films yields a plateau layer thickness ${ }^{6,8-10,18,51-56}$ and that exchange between adsorbed polymer chains and overlaying unadsorbed chains is negligible under typical working conditions of polymer/substrate systems., ${ }^{9,57}$ Adsorbed nanolayers of pyrene-labeled PS atop isolated NPs can be directly characterized via TEM imaging and fluorescence to measure layer thickness and $T_{g}$, respectively. Furthermore, the isolated NPs can also be redispersed into a matrix of neat PS to yield nanocomposites wherein pyrene-labeled PS is isolated within the irreversibly adsorbed layer at the NP/matrix interface. Thus, fluorescence spectroscopy of this unique experimental system enables direct in situ measurement of adsorbed layer $T_{\mathrm{g}}$ in polymer nanocomposites.

Successful implementation of the procedure is ensured by two critical features: (i) good dispersion of NPs within their initial polymer matrix to ensure uninhibited formation of adsorbed layers during annealing and (ii) removal of NPs bearing adsorbed layers from the nanocomposites. Here, we prepared nanocomposites following a previously reported protocol. ${ }^{19}$ In brief, $\mathrm{SiO}_{x} \mathrm{NPs}\left(d_{\mathrm{NP}}=289 \pm 12 \mathrm{~nm}\right)$ were prepared via the Stöber method and transferred from the aqueous reaction medium into DMF. The resulting $\mathrm{SiO}_{x} / \mathrm{DMF}$ dispersion was combined with pyrene-labeled PS to produce viscous slurry, which was subsequently drop-cast onto a glass substrate and annealed above $T_{\mathrm{g}}$ under vacuum for a specified amount of time designated $t_{\text {ann }}$, producing well-dispersed nanocomposites which serve as a platform for adsorption. We isolated NPs bearing irreversibly adsorbed layers of pyrenelabeled PS from unadsorbed matrix chains by dissolving the nanocomposites in toluene for $30 \mathrm{~min}$ under continuous sonication. Subsequently, the NPs were separated from the solution and redispersed into fresh toluene, marking the end of the first of five washing cycles. To confirm complete removal of unadsorbed pyrene-labeled PS from the NPs, we performed fluorescence spectroscopy on the supernatant. As shown in Figure $1 \mathrm{~b}$, after four wash cycles, the supernatant contained no detectable amount of pyrene-labeled PS, rendering it indistinguishable from the blank substrate and thus confirming the absence of the dissolved polymer in the solution. Figure $1 c, d$ provides representative images of isolated NPs bearing adsorbed layers and NPs redispersed in a new matrix, respectively.

Adsorbed Layer Thickness and Growth. To observe the growth of irreversibly adsorbed layers atop the NPs, we prepared nanocomposites under various annealing times $\left(t_{\mathrm{ann}}\right)$ at bulk PS $T_{\mathrm{g}}+50 \mathrm{~K}\left(\sim 156{ }^{\circ} \mathrm{C}\right)$ and subsequently isolated the NPs following the procedure outlined above. Ellipsometry is the conventional method for measuring adsorbed layer thickness in thin films but is incompatible with detailed characterization of NPs. However, the formation of such layers atop NPs offers a unique opportunity to visualize their growth as a function of time using TEM. Figure 2 shows TEM images of NPs isolated from nanocomposites annealed at $t_{\mathrm{ann}}=0,1,3$, and $10 \mathrm{~h}$. No adsorbed layers were observed in NPs prepared under $t_{\mathrm{ann}}=0 \mathrm{~h}$; however, NPs for which $t_{\mathrm{ann}}>0 \mathrm{~h}$ featured clearly visible polymer coatings distinguishable as irreversibly adsorbed layers of pyrene-labeled PS. Similar to thin films, the thickness of the adsorbed layer exhibited a strong dependence on $t_{\mathrm{ann}}$, as shown in Figure 2.

We further confirmed the formation and stability of irreversibly absorbed layers atop the NPs via BLS, a labelfree, contactless, nondestructive technique which probes highfrequency NP elastic vibrations. ${ }^{49}$ In accordance with previous studies of soft core-thin shell polymer NPs, BLS measurements of colloidal clusters of $\mathrm{PS} / \mathrm{SiO}_{x}$ shell-core NPs indicated that the quadrupolar $(1,2)$ mode $^{58,59}$ - denoting shearing motion on the NP surface-was sensitive to NP surface dynamics. Figure 3 a shows the evolution of the BLS $I(f)$ spectrum as a function of $t_{\text {ann }}$. Longer $t_{\text {ann }}$ elicited a marked broadening and split of the $(1,2)$ mode (Figure $3 a)$, as well as the emergence of the translation-like $(1,1)$ mode (see Figure S3 in the Supporting Information). Note that the latter is interaction-induced ${ }^{49}$ and therefore absent in the pristine $\mathrm{SiO}_{x} \mathrm{NPs}$, which exhibit negligible interparticle adhesion and consequently feature only the $(1,2)$ mode (see Figure $3 a$ at $t_{\mathrm{ann}}=0 \mathrm{~h}$ ). Taken together, these attributes are strong evidence of surface modification via the PS adsorption. Representation of the main $(1,2)$ mode by double Gaussian (Figure 3a) revealed progressively increased splitting of the two frequencies $\left(f_{1}<f_{2}\right)$ with $t_{\text {ann }}$. Calculated effective $f_{\mathrm{L}}=2 f_{1}-f_{2}$, which corresponds to the $(1,2)$ mode of individual NPs, exhibits decreasing frequency with $t_{\text {ann }}$ and saturates near $t_{\text {ann }} \sim 10 \mathrm{~h}$ (see Figure S4a in the Supporting Information), further supporting the $t_{\mathrm{ann}}$ dependence of adsorbed layer thickness. Finite element modeling similarly attributed the $t_{\mathrm{ann}}$-dependent decrease in $f_{\mathrm{L}}$ to the concurrent increase in adsorbed PS layer 

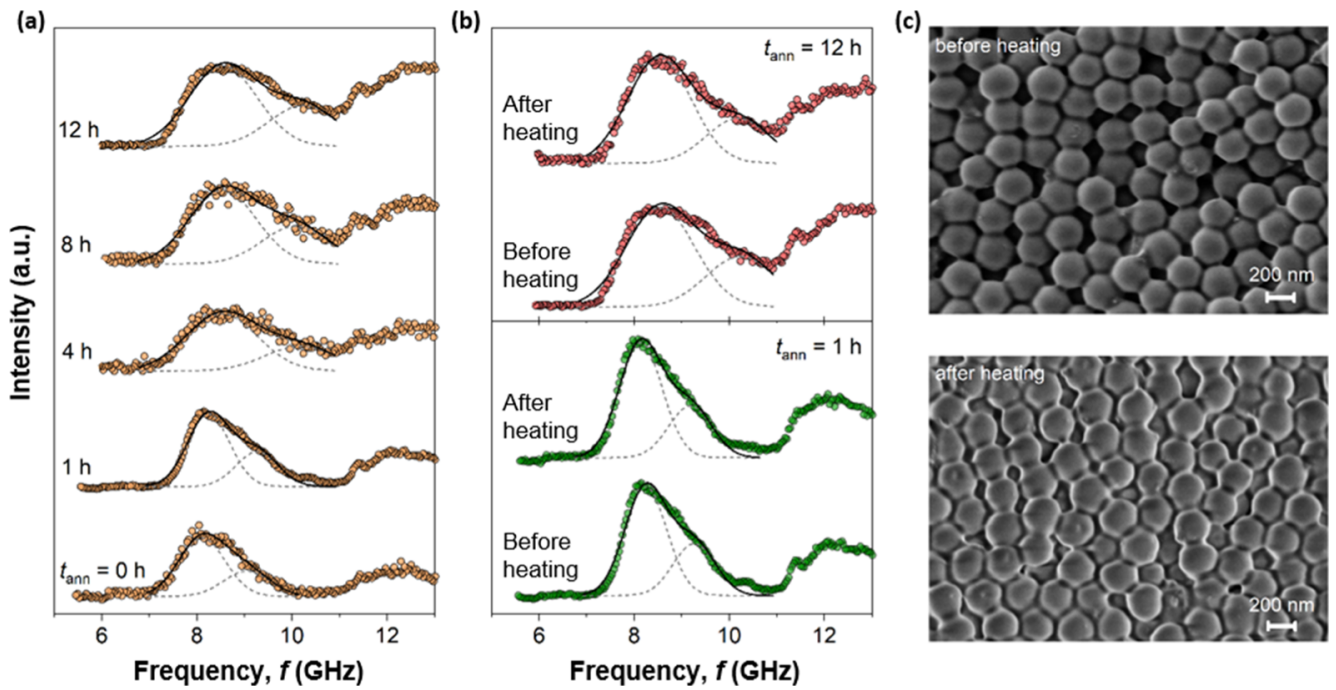

Figure 3. (a) BLS spectra of NPs bearing PS-adsorbed layers corresponding to different $t_{\text {ann }}$. Black solid lines represent the fundamental $(1,2)$ elastic vibration mode of clustered NPs bearing adsorbed layers. Double Gaussian decomposition (gray dashed lines) reveals $t_{\text {ann }}$-dependent splitting of $f_{1}$ and $f_{2}$. (b) BLS spectra of respective NP samples before and after heating to $T=T_{\mathrm{g}}+50 \mathrm{~K}-156^{\circ} \mathrm{C}$ for NPs bearing adsorbed layers of PS corresponding to $t_{\mathrm{ann}}=12 \mathrm{~h}$ (upper panel) and $1 \mathrm{~h}$ (lower panel). (c) SEM images of NPs bearing PS-adsorbed layers before (top) and after (bottom) heating.

thickness (see Figure $\mathrm{S} 4 \mathrm{~b}$ in the Supporting Information). Altogether, the BLS data in Figures 3a and S4 corroborate the conclusions of our TEM investigation (Figure 2), which revealed the adsorbed layer structure to be $t_{\mathrm{ann}}$-dependent. Moreover, BLS measurements verified the thermal stability of the PS-adsorbed layers, as the characteristic vibrational mode of each respective sample was essentially unchanged following heat treatment (Figure 3b). Imaging before and after hightemperature annealing, depicted in Figure 3c, likewise revealed no considerable differences in the adsorbed layer structure, thereby authenticating that the nature of polymer adsorption atop the nanoparticles was irreversible.

To quantify the dependence of absorbed layer thickness $\left(h_{\mathrm{ads}}\right)$ on annealing time, Figure 4 plots $h_{\mathrm{ads}}$ versus $t_{\mathrm{ann}}$ for

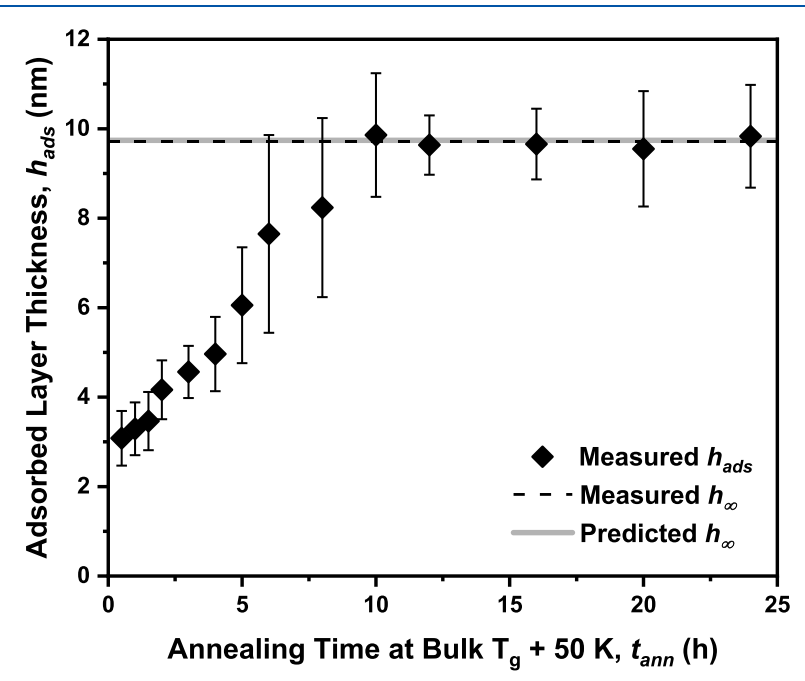

Figure 4. Adsorbed layer thickness as a function of $t_{\mathrm{ann}}$ for pyrenelabeled PS. Thickness was measured under TEM. Error bars represent \pm 1 standard deviation for $h_{\text {ads }}$ of identically prepared samples. The dashed line shows equilibrium thickness $h_{\infty}$ obtained by averaging all $h_{\text {ads }}$ for which $t_{\text {ann }}$ was $10 \mathrm{~h}$ or greater. The solid line is the $h_{\infty}$ predicted by the literature-reported scaling relationship. ${ }^{61}$ irreversibly adsorbed layers prepared by annealing at $T_{\mathrm{g}}+50$ $\mathrm{K}$. Adsorbed layer thickness initially grows linearly with time, but for $t_{\mathrm{ann}} \geq 10 \mathrm{~h}$, it is time-independent. The growth curve reflects the rapid coverage of the nanoparticle surface at short annealing times, followed by little to no additional growth as the previously adsorbed polymer physically impedes the adsorption of subsequent chains. Observations of rapid initial growth of PS layers on the NPs are consistent with the proposed kinetic model accounting for surface coverage, ${ }^{8,56}$ as well as growth trends reported for irreversibly adsorbed layers of PS in analogous thin-film experiments. ${ }^{9,18,55}$ The equilibrium thickness $\left(t_{\mathrm{ann}} \geq 10 \mathrm{~h}\right)$ of the adsorbed layer was measured to be $9.7 \pm 1.1 \mathrm{~nm}$, consistent with the predicted equilibrium thickness of $9.7 \mathrm{~nm}$ estimated on the basis of PS $R_{\mathrm{g}}$ in $\mathrm{THF}^{60}$ (a good solvent for PS, comparable to DMF) and the linear scaling relationship $h_{\infty}=0.47 R_{\mathrm{g}}$ for PS adsorption upon a $\mathrm{SiO}_{x}$ surface. ${ }^{61}$

$T_{\mathrm{g}}$ of Adsorbed Layers atop Isolated NPs. The $T_{\mathrm{g}}$ of irreversibly adsorbed layers atop isolated NPs was measured by steady-state fluorescence spectroscopy, a well-established technique for directly measuring local glassy-state properties of dye molecules preferentially located within a heterogenous system. ${ }^{39}$ Following excitation, dye molecules undergo deexcitation via competing mechanisms: nonradiative processes restricted by local density and processes which produce fluorescence. As a result, the fluorescence emission spectra of pyrene-labeled PS atop the isolated NPs are strongly governed by temperature, as depicted in the inset of Figure 5a. Importantly, the temperature dependence of the integrated spectral intensity exhibits a marked change at the glass transition, consistent with dilatometry. As shown in Figure 5a, $T_{\mathrm{g}}$ is defined as the intersection of linear fits of the glassy and rubbery state lines. ${ }^{43,62,63}$ Fluorescence measurements revealed that local $T_{\mathrm{g}}$ of adsorbed layers atop the isolated NPs was correlated to layer thickness. As shown in Figure 5b, adsorbed layers with smaller $h_{\text {ads }}$ featured correspondingly lower adsorbed layer $T_{\mathrm{g}}$.

Taken together with the $t_{\mathrm{ann}}$ dependence of $h_{\mathrm{ads}}$ (Figure 4), the positive correlation of $h_{\mathrm{ads}}$ versus $T_{\mathrm{g}}$ in Figure $5 \mathrm{~b}$ suggests 
(a)

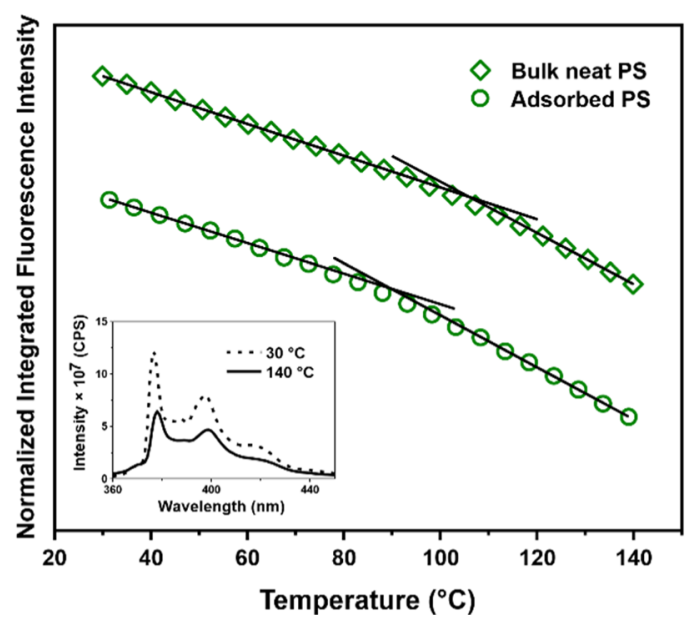

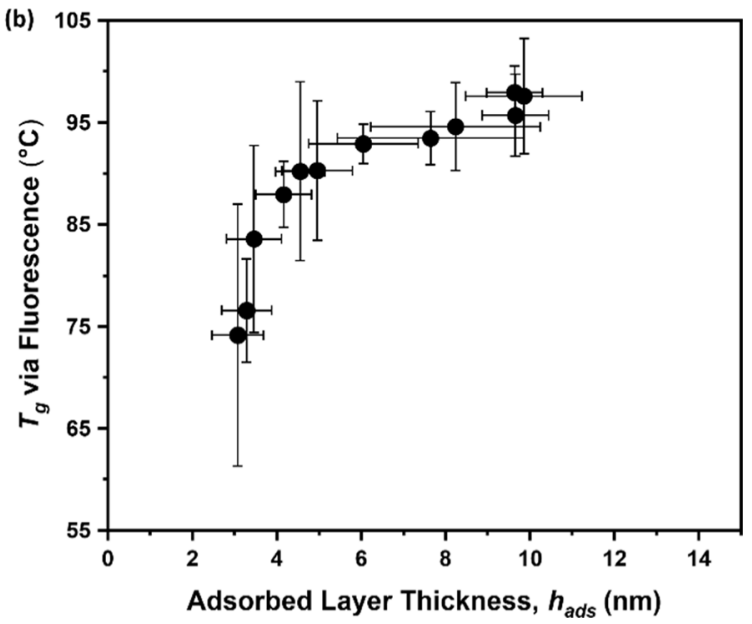

Figure 5. (a) Representative $T_{\mathrm{g}}$ measurement of pyrene-labeled PS in bulk and in the adsorbed layer geometry. Integrated intensities are normalized and shifted vertically for clarity. Inset: emission spectra of pyrene-labeled PS at two different temperatures. (b) Plot of absorbed layer $T_{g}$ atop isolated NPs vs $h_{\text {ads }}$. Error bars represent \pm 1 standard deviation for identically prepared adsorbed layers atop NPs.

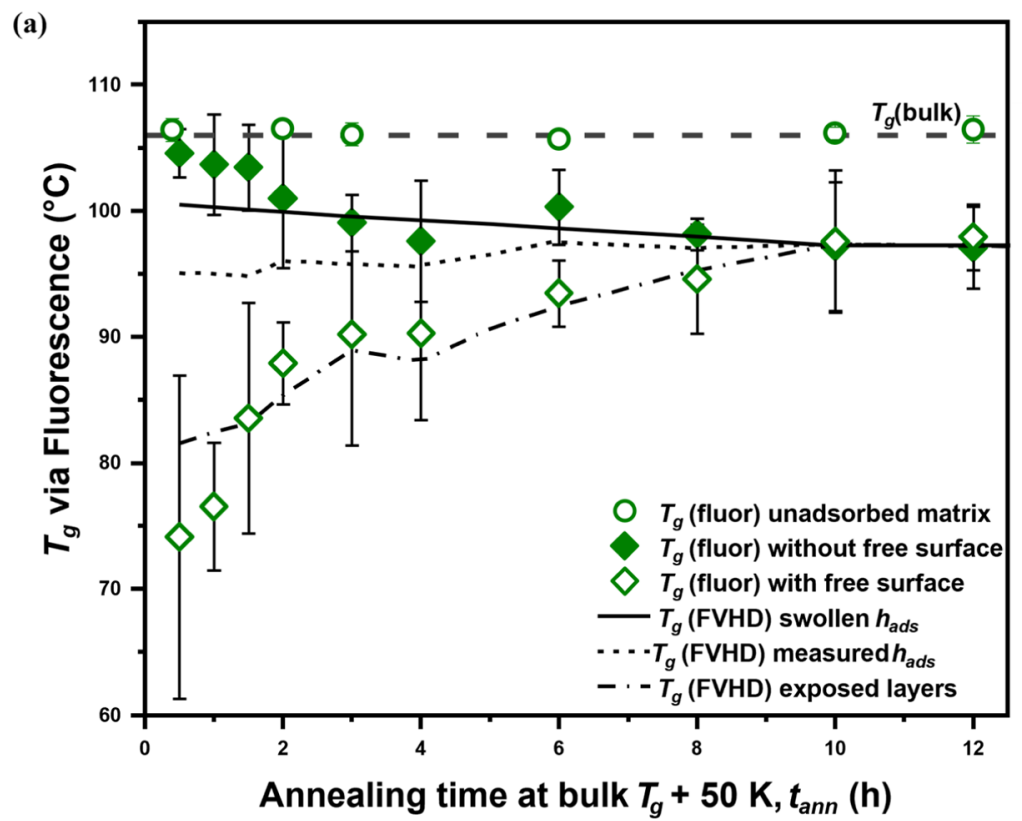

(b)
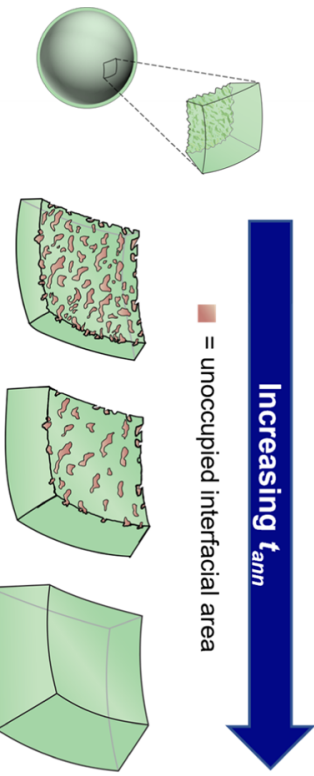

Figure 6. (a) $T_{\mathrm{g}}$ of adsorbed layers at various stages of development (i.e., $t_{\mathrm{ann}}$ ) atop isolated NPs and NPs redispersed within polymer nanocomposites. Also shown are complementary $T_{\mathrm{g}}$ measurements of the unadsorbed matrices of nanocomposites. Each data point represents the average of at least three independent measurements, and the error bars represent \pm 1 standard deviation of $T_{\mathrm{g}}$ for identically prepared samples. Error bars are smaller than some data points. $T_{\mathrm{g}}$ data were fitted to the FVHD model, see the Supporting Information. (b) Schematic depiction of adsorbed layer growth atop NPs, with tan-shaded regions denoting unoccupied interface. (Note that for isolated NPs, i.e., those not redispersed in a matrix, the unoccupied interface also includes the outer free interface.) Longer $t_{\text {ann }}$ elicits the increasingly filled NP/adsorbed layer interface and greater layer thickness, both of which contribute to the partial recovery of bulk $T_{\mathrm{g}}$.

that adsorbed layer $T_{\mathrm{g}}$ evolves with annealing time. Indeed, we observed that the evolution of adsorbed layer $T_{\mathrm{g}}$ atop the isolated NPs was roughly proportional-at least initially-to $t_{\text {ann }}$ as shown in Figure 6a; see open diamonds. The average $T_{\mathrm{g}}$ of layers corresponding to short adsorption times was significantly depressed-as much as $30 \mathrm{~K}$-relative to bulk PS. With an increase in adsorption time, the adsorbed layer $T_{\mathrm{g}}$ increased toward the bulk value. This evolution reflects the decreasing contribution of the free surface in governing the $T_{\mathrm{g}}$ of isolated absorbed layers atop NPs, which we attribute to two factors: (i) the free surface comprises a smaller proportion of the adsorbed layer volume with increasing thickness and (ii) density of the adsorbed layer increases as additional chain adsorption occurs.

Notably, the $T_{\mathrm{g}}$ of isolated adsorbed layers atop the NPs never fully recovered the bulk $T_{g}$, ultimately plateauing at 97.4 $\pm 3.7{ }^{\circ} \mathrm{C}$ instead. This finding is in excellent qualitative agreement with the partial recovery of $T_{\mathrm{g}}$ reported for analogous thin-film adsorption experiments, ${ }^{9}$ which is not surprising considering the relative ratio of the polymer $R_{\mathrm{g}}$ to the diameter of the NPs in this study. Intriguingly, the $t_{\mathrm{ann}}$ dependence of the isolated adsorbed layer $T_{\mathrm{g}}$ resembles that of the corresponding $h_{\mathrm{ads}}$, as both quantities become independent of the annealing time at $t_{\mathrm{ann}} \sim 10 \mathrm{~h}$. This congruence not only evidences the interrelatedness of $t_{\mathrm{ann}}, h_{\mathrm{ads}}$, and $T_{\mathrm{g}}$ but also 
reinforces that variability in routine processing conditions can exert outsize influence in determining end properties.

Local $T_{\mathrm{g}}$ of Adsorbed Layers in Nanocomposites. To gain insights into how irreversible adsorption impacts interfacial properties of real nanocomposites used for practical applications, the isolated NPs bearing pyrene-labeled irreversibly adsorbed layers were redispersed in an unlabeled PS matrix, yielding a polymer nanocomposite. Because the pyrene molecules were located solely within the irreversibly adsorbed layers atop the NPs and chain exchange between labeled and unlabeled PS is negligible, ${ }^{9,57}$ fluorescence spectroscopy acquired $T_{\mathrm{g}}$-related information from this region only, affording in situ measurement of the local $T_{\mathrm{g}}$ at the adsorbed interface. As shown in Figure 6a (see filled diamonds), the average $T_{\mathrm{g}}$ of irreversibly adsorbed layers atop the redispersed NPs was depressed compared to the bulk $T_{g}$, albeit not to a large extent for layers grown under short annealing times. For instance, at $t_{\mathrm{ann}} \sim 0.5 \mathrm{~h}$, the observed $T_{\mathrm{g}}$ was only $1.5 \pm 1.9 \mathrm{~K}$ lower than, and therefore within the error of, the bulk value. Beyond short annealing times, the $T_{\mathrm{g}}$ of in situ NPs generally decreased with increasing $t_{\mathrm{ann}}$ until stabilizing when $t_{\mathrm{ann}} \geq 10 \mathrm{~h}$. Comparing the $T_{\mathrm{g}}$ of adsorbed layers of the corresponding $t_{\mathrm{ann}}$ atop analogous isolated and redispersed NPs indicated that free surface effects ${ }^{64}$ dominated at short adsorption times, as the presence of a matrix induced near complete recovery of bulk $T_{\mathrm{g}}$. The degree of recovery-and hence the effect of the free interface-shrinks until disappearing completely at $t_{\text {ann }} \geq$ $10 \mathrm{~h}$, where it can be deduced that the chain density and degree of anchoring are sufficient to restrict the enhanced mobility typical of the free interface. As such, perturbed properties associated with this timeframe can be attributed solely to the effects of irreversible adsorption.

A mechanistic interpretation of the perturbed $T_{\mathrm{g}}$ properties - namely, reduction of adsorbed layer $T_{\mathrm{g}}$-is afforded by the FVHD model, which proposes that the underlying mechanism eliciting reduced $T_{\mathrm{g}}$ at a polymer/substrate interface is the diffusion of free volume holes out of the matrix via a free interface, which can occur at a "true" (i.e., air/ polymer) free interface or at unoccupied spaces at a polymer/ substrate interface, where there is excess free volume. ${ }^{9,65,66}$ The FVHD model has been adopted to describe the enhanced aging observed within $\mathrm{PMMA} / \mathrm{SiO}_{x}$ nanocomposites, ${ }^{13}$ albeit without regard to local heterogeneities, but rather according to average aging properties throughout the system.

For the purposes of evaluating our polymer nanocomposite system, any roughness present on the $\mathrm{SiO}_{x}$ surface was neglected, and adsorbed layer growth was considered to proceed outward radially in an essentially even front, an assumption supported by TEM imaging (Figure 2). As PS $R_{\mathrm{g}}$ $\ll d_{\mathrm{NP}}$, curvature effects were assumed to be negligible in our calculations. This framework entails that annealing causes unoccupied spaces at the NP/adsorbed PS interface, depicted by the tan-shaded regions in Figure $6 \mathrm{~b}$, to progressively fill with adsorbed chains. In an isolated NP, the free surface at the adsorbed layer/air interface remains available as a sink for free volume during the evolution of the adsorbed layer. However, the $t_{\mathrm{ann}}$-driven progression of chain adsorption at the adsorbed layer/NP interface coincides with the corresponding increase in chain density. Increasing density with $t_{\text {ann }}$ elicits progressively increasing $T_{\mathrm{g}}$ until the adsorbed layer/NP interface is completely filled, at which point $T_{\mathrm{g}}$ stabilizes. Accordingly, eq 1 (see the Supporting Information for derivation) describes the $t_{\mathrm{ann}}$-dependent rate of free volume changes within adsorbed layers atop the isolated NPs

$$
\frac{A_{\text {unoccupied }}}{A_{\text {total }}}=\left(\frac{A_{\text {unoccupied }}}{A_{\text {total }}}\right)_{\max }-a \cdot t_{\text {ann }}
$$

Here, $t_{\mathrm{ann}}$ is the adsorption time; $A_{\text {total }}$ is the summed area of the adsorbed layer/NP interface and adsorbed layer/free interface; $A_{\text {unoccupied }}$ is the summed area of the unoccupied interface at both the adsorbed layer/NP interface and the adsorbed layer/free interface; $\left(A_{\text {unoccupied }} / A_{\text {total }}\right)_{\max }$ is the proportional amount of the unoccupied interface at $t_{\mathrm{ann}}=0$; and $a$ is the rate of unoccupied interface filling. An isolated nanoparticle with a fully developed adsorbed layer-that is, an adsorbed layer of equilibrium thickness-is completely filled with adsorbed chains at the adsorbed layer/NP interface, while the adsorbed layer/air interface remains completely unoccupied. Accounting for the boundary condition that the onset of equilibrium $T_{\mathrm{g}}$ in this system corresponds to $A_{\text {unoccupied }} / A_{\text {total }}=$ 0.5 in the absence of curvature effects, we determined that adsorbed layer $T_{\mathrm{g}}$ corresponding to various layer thicknesses atop the isolated NPs was governed by an interfacial filling rate of $a=0.019 \mathrm{~h}^{-1}$ (see the Supporting Information).

By reconceptualizing the adsorbed layer/free interface as an adsorbed layer/matrix interface, we can consider NPs redispersed in a polymer matrix. In this system, the unoccupied area is contributed only by the NP/adsorbed layer interface, as interpenetration of the surrounding matrix eliminates the unoccupied area at the adsorbed layer/matrix interface. Accordingly, a description of $t_{\mathrm{ann}}$-dependent free volume changes for redispersed NPs is given by eq 2

$$
\frac{A_{\text {unoccupied }}}{A_{\text {total }}}=\left(\frac{A_{\text {unoccupied }}}{A_{\text {total }}}\right)_{\min }+b \cdot t_{\text {ann }}
$$

Here, $A_{\text {total }}$ is the same as in eq 1 , but $A_{\text {unoccupied }}$ represents contributions from only the adsorbed layer/NP interface. Increased chain adsorption with the annealing time progressively inhibits matrix interpenetration of the adsorbed layer, such that unoccupied spaces at the NP/adsorbed layer interface are more readily occupied by penetrating matrix chains at shorter adsorption times. Therefore, the term $\left(A_{\text {unoccupied }} / A_{\text {total }}\right)_{\min }$ is the proportional amount of unoccupied interface at $t_{\mathrm{ann}}=0$ and $b$ refers to the interfacial filling rate of unoccupied area, $b=0.031 \mathrm{~h}^{-1}$ (see the Supporting Information). The interfacial filling rates calculated using FVHD theory in eqs 1 and 2 correspond to ( $A_{\text {unoccupied }}$ / $\left.A_{\text {total }}\right)_{\max }=0.69$, suggesting that at $t_{\mathrm{ann}}=0$, the NP/adsorbed layer interface of an isolated nanoparticle contributes over onethird $(\sim 38 \%)$ of the unoccupied area for outward diffusion of free volume holes. (In a redispersed nanoparticle, this contribution is essentially $100 \%$ as the surrounding matrix eliminates the unoccupied area at the adsorbed layer/matrix interface. $)$ Notably, our fitted value of $\left(A_{\text {unoccupied }} / A_{\text {total }}\right)_{\max }$ for an isolated nanoparticle is significantly greater than that deduced from analogous PS adsorption in the exposed thin film regime, ${ }^{9}$ indicating that the substrate interface is a more significant source of interfacial free volume in our nanocomposite system. We note that this may reflect neglected curvature effects or differences in substrate properties due to some degree of porosity characteristic of Stöber silica. However, we also highlight this discrepancy as an illustrative opportunity for future application of our model system to 
elucidate a better fundamental understanding of polymer adsorption than conventional approaches have historically allowed.

Despite quantitative differences in fitting parameters, the good qualitative agreement between our nanocomposite experiments and the thin film experiments ${ }^{9}$ suggests a unifying governing mechanism for $T_{\mathrm{g}}$ of adsorbed layers in both systems. This mechanism entails a $t_{\mathrm{ann}}$-dependent increase in chain density in the adsorbed layers, which is illustrated in Figure $6 \mathrm{~b}$. As the adsorbing surface is progressively filled with chains, the contrast in the segmental densities of the adsorbed layer and the surrounding matrix decreases. Consequently, removal of the matrix reveals that with increasing $t_{\mathrm{ann}}, T_{\mathrm{g}}$ of the ex situ adsorbed layers shifts toward the bulk value. However, while additional in situ adsorption increases adsorbed chain density, it also incrementally impedes interpenetration of the surrounding matrix for further adsorption, eventually manifesting in a degree of packing frustration adequate to obstruct adsorption entirely. At this point, regions of excess free volume still exist within the adsorbed layer, but they are inaccessible by free matrix chains, accounting for the merely partial recovery of bulk $T_{\mathrm{g}}$ in ex situ adsorbed layers prepared from long-annealed (in this study, $t_{\mathrm{ann}}>10 \mathrm{~h}$ ) samples; see Figure 6a. Meanwhile, for the case of adsorbed layers within nanocomposites, the presence of an adjacent matrix facilitates nearly complete recovery of bulk $T_{\mathrm{g}}$ in short-annealed samples due to relatively unrestricted access to the minimally dense adsorbed layer by surrounding chains, but in long-annealed samples, the enhanced density of adsorbed chains is offset by the less effective interpenetration by matrix chains, giving rise to a consistently reduced $T_{\mathrm{g}}$ compared to the bulk value. In other words, once adsorption has proceeded to the point that packing frustration completely inhibits subsequent chain adsorption, the presence of a matrix overlayer is inconsequential, such that long-annealed adsorbed layer $T_{\mathrm{g}}$ is consistent across ex situ and nanocomposite samples. This implies that the discrepancy between bulk $T_{\mathrm{g}}$ and equilibrium adsorbed layer $T_{\mathrm{g}}$ is attributable to the effects of irreversible adsorption alone.

Insensitivity of Matrix $T_{\mathrm{g}}$ to Perturbations Originating from PS-Adsorbed Layers. To understand how dynamic perturbations manifesting from adsorbed layers at the polymer $/ \mathrm{SiO}_{x}$ interface impact the matrix properties of the nanocomposite, we devised a different, yet complementary, scheme, as shown in Figure 7. This scheme produced polymer nanocomposites in which fluorescent labels were preferentially located on polymer chains solely within the free matrix rather

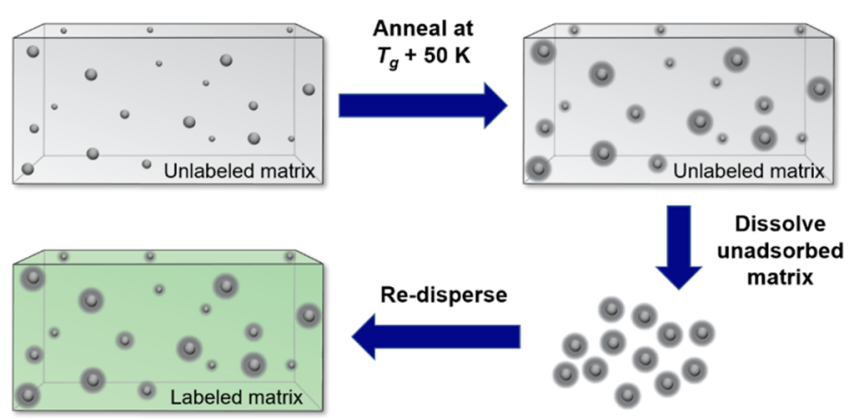

Figure 7. Procedure to prepare polymer nanocomposites in which fluorescent dyes are isolated within the unadsorbed matrix rather than the irreversibly adsorbed layers. than within regions irreversibly adsorbed atop the NP surface. For this particular PS/SiO ${ }_{x}$ nanocomposite, the $T_{\mathrm{g}}$ within the unadsorbed matrix region approximated bulk $T_{g}$, regardless of adsorbed layer thickness at the NPs (Figure 6a; see open circles). This finding is seemingly consistent with a range of studies which report PS $T_{\mathrm{g}}$ to be generally unperturbed at nonfree interfaces. ${ }^{20,40,43,67,68}$ It may also be viewed within the framework of simulated predictions that the matrix is shielded from the dynamic perturbations of NPs, given that they are encased in a layer of bound-that is, essentially irreversibly adsorbed-polymer. ${ }^{69}$ While PS and silica are not generally considered to comprise a strongly interacting pair, the chain anchoring and reduced conformational entropy imparted by irreversible adsorption may contribute to a local environment resembling that of a strongly interacting bound layer in some respect which is key for governing how perturbations propagate throughout a polymer system.

\section{CONCLUSIONS}

We have demonstrated a model system for the direct measurement of local properties arising from the effects of irreversible adsorption in polymer nanocomposites. Work in polymer nanocomposites has historically ignored this parameter, despite its ubiquity in high-temperature annealed samples and the possibility for incomplete growth of adsorbed layers to produce variable local environments. Accordingly, the approach demonstrated herein can serve as a useful platform to extend our current understanding of structure-processingproperty relationships in systems featuring nanoconfinement. We have reported the initial application of our approach to study the glassy properties of a $\mathrm{PS} / \mathrm{SiO}_{x}$ nanocomposite, wherein local $T_{\mathrm{g}}$ of adsorbed regions was observed to be (1) annealing time-dependent, (2) consistently depressed compared to the bulk PS $T_{\mathrm{g}}$, and (3) increasingly less influenced by the presence of a bulk matrix overlayer as adsorbed layers grew thicker. These findings are consistent with established studies of adsorbed PS thin films ${ }^{9}$ and therefore support the validity of our model system and approach for generalized study of the local perturbations attributable to irreversible adsorption at nanoparticle interfaces. Further application of our model system may enable new insights into these perturbations and reveal how they may be leveraged to design useful bulk properties in polymer nanocomposites.

\section{ASSOCIATED CONTENT}

\section{SI Supporting Information}

The Supporting Information is available free of charge at https://pubs.acs.org/doi/10.1021/acs.macromol.1c01557.

Characterization and simulation details and the fitting procedure for the FVHD model (PDF)

\section{AUTHOR INFORMATION}

\section{Corresponding Authors}

Biao Zuo - Department of Chemical and Biological Engineering, Princeton University, Princeton, New Jersey 08544, United States; Department of Chemistry, Key Laboratory of Surface \& Interface Science of Polymer Materials of Zhejiang Province, Zhejiang Sci-Tech University, Hangzhou 310018, China; (1) orcid.org/0000-0002-49218823; Email: chemizuo@zstu.edu.cn

Rodney D. Priestley - Department of Chemical and Biological Engineering and Princeton Institute for the Science 
and Technology of Materials, Princeton University, Princeton, New Jersey 08544, United States; (1) orcid.org/0000-00016765-2933; Email: rpriestl@princeton.edu

\section{Authors \\ Katelyn Randazzo - Department of Chemical and Biological Engineering, Princeton University, Princeton, New Jersey 08544, United States \\ Malgorzata Bartkiewicz - Max Planck Institute for Polymer Research, Mainz 55128, Germany \\ Bartlomiej Graczykowski - Faculty of Physics, Adam Mickiewicz University, Poznan 61-614, Poland \\ Daniele Cangialosi - Centro de Física de Materiales (CSIC- UPV/EHU), San Sebastián 20018, Spain; Donostia International Physics Center (DIPC), San Sebastián 20018, Spain \\ George Fytas - Max Planck Institute for Polymer Research, Mainz 55128, Germany; (1) orcid.org/0000-0003-2504- 6374}

Complete contact information is available at:

https://pubs.acs.org/10.1021/acs.macromol.1c01557

\section{Notes}

The authors declare no competing financial interest.

\section{ACKNOWLEDGMENTS}

The authors acknowledge the use of Princeton's Imaging and Analysis Center, which is partially supported by the Princeton Center for Complex Materials, a National Science Foundation (NSF)-MRSEC program (DMR-2011750). K.R. acknowledges the support of the National Science Foundation Graduate Research Fellowship Program under grant no. DCE-1656466. M.B. and G.F. acknowledge the financial support from ERC AdG SmartPhon (grant no. 694977). M.B. and G.F. acknowledge the financial support of the Foundation for Polish Science (POIR.04.04.00-00-5D1B/18). B.Z. acknowledges the financial support from the Natural Science Foundation of China (grant nos. 22122306, 21973083, and 22011530456).

\section{REFERENCES}

(1) Hashemi, A.; Jouault, N.; Williams, G. A.; Zhao, D.; Cheng, K. J.; Kysar, J. W.; Guan, Z.; Kumar, S. K. Enhanced Glassy State Mechanical Properties of Polymer Nanocomposites via Supramolecular Interactions. Nano Lett. 2015, 15, 5465-5471.

(2) Maillard, D.; Kumar, S. K.; Fragneaud, B.; Kysar, J. W.; Rungta, A.; Benicewicz, B. C.; Deng, H.; Brinson, L. C.; Douglas, J. F. Mechanical Properties of Thin Glassy Polymer Films Filled with Spherical Polymer-Grafted Nanoparticles. Nano Lett. 2012, 12, 39093914.

(3) Guo, Z.; Kim, T. Y.; Lei, K.; Pereira, T.; Sugar, J. G.; Hahn, H. T. Strengthening and thermal stabilization of polyurethane nanocomposites with silicon carbide nanoparticles by a surface-initiatedpolymerization approach. Compos. Sci. Technol. 2008, 68, 164-170.

(4) Jia, X.; Li, Y.; Cheng, Q.; Zhang, S.; Zhang, B. Preparation and properties of poly(vinyl alcohol)/silica nanocomposites derived from copolymerization of vinyl silica nanoparticles and vinyl acetate. Eur. Polym. J. 2007, 43, 1123-1131.

(5) Everett, D. H. Enthalpy and entropy effects in adsorption from solution. J. Phys. Chem. 1981, 85, 3263-3265.

(6) Napolitano, S.; Wübbenhorst, M. The lifetime of the deviations from bulk behaviour in polymers confined at the nanoscale. Nat. Commun. 2011, 2, 260.

(7) Napolitano, S.; Capponi, S.; Vanroy, B. Glassy dynamics of soft matter under $1 \mathrm{D}$ confinement: how irreversible adsorption affects molecular packing, mobility gradients and orientational polarization in thin films. Eur. Phys. J. E 2013, 36, 61.

(8) Housmans, C.; Sferrazza, M.; Napolitano, S. Kinetics of Irreversible Chain Adsorption. Macromolecules 2014, 47, 3390-3393.

(9) Burroughs, M. J.; Napolitano, S.; Cangialosi, D.; Priestley, R. D. Direct Measurement of Glass Transition Temperature in Exposed and Buried Adsorbed Polymer Nanolayers. Macromolecules 2016, 49, 4647-4655.

(10) Zuo, B.; Zhou, H.; Davis, M. J. B.; Wang, X.; Priestley, R. D. Effect of Local Chain Conformation in Adsorbed Nanolayers on Confined Polymer Molecular Mobility. Phys. Rev. Lett. 2019, 122, 217801.

(11) DeMaggio, G. B.; Frieze, W. E.; Gidley, D. W.; Zhu, M.; Hristov, H. A.; Yee, A. F. Interface and Surface Effects on the Glass Transition in Thin Polystyrene Films. Phys. Rev. Lett. 1997, 78, 15241527.

(12) Napolitano, S.; Wübbenhorst, M. Dielectric Signature of a Dead Layer in Ultrathin Films of a Nonpolar Polymer. J. Phys. Chem. B 2007, 111, 9197-9199.

(13) Cangialosi, D.; Boucher, V. M.; Alegría, A.; Colmenero, J. Free volume holes diffusion to describe physical aging in poly(mehtyl methacrylate)/silica nanocomposites. J. Chem. Phys. 2011, 135, 014901.

(14) Kourkoutsaki, T.; Logakis, E.; Kroutilova, I.; Matejka, L.; Nedbal, J.; Pissis, P. Polymer dynamics in rubbery epoxy networks/ polyhedral oligomeric silsesquioxanes nanocomposites. J. Appl. Polym. Sci. 2009, 113, 2569-2582.

(15) Nadgorny, M.; Gentekos, D. T.; Xiao, Z.; Singleton, S. P.; Fors, B. P.; Connal, L. A. Manipulation of Molecular Weight Distribution Shape as a New Strategy to Control Processing Parameters. Macromol. Rapid Commun. 2017, 38, 1700352.

(16) Gupta, S. S.; Solanki, N.; Serajuddin, A. T. M. Investigation of Thermal and Viscoelastic Properties of Polymers Relevant to Hot Melt Extrusion, IV: Affinisol HPMC HME Polymers. AAPS PharmSciTech 2016, 17, 148-157.

(17) Müller, C. On the Glass Transition of Polymer Semiconductors and Its Impact on Polymer Solar Cell Stability. Chem. Mater. 2015, 27, 2740-2754.

(18) Davis, M. J. B.; Randazzo, K.; Zuo, B.; Priestley, R. D. Revealing Interfacial Interactions in Random Copolymer Adsorbed Layers by Solvent Leaching. Macromol. Rapid Commun. 2020, 41, 1900582.

(19) Meth, J. S.; Zane, S. G.; Chi, C.; Londono, J. D.; Wood, B. A.; Cotts, P.; Keating, M.; Guise, W.; Weigand, S. Development of Filler Structure in Colloidal Silica-Polymer Nanocomposites. Macromolecules 2011, 44, 8301-8313.

(20) Wei, T.; Torkelson, J. M. Molecular Weight Dependence of the Glass Transition Temperature (Tg)-Confinement Effect in WellDispersed Poly(2-vinyl pyridine)-Silica Nanocomposites: Comparison of Interfacial Layer Tg and Matrix Tg. Macromolecules 2020, 53, $8725-8736$.

(21) Zhang, W. D.; Shen, L.; Phang, I. Y.; Liu, T. Carbon Nanotubes Reinforced Nylon-6 Composite Prepared by Simple Melt-Compounding. Macromolecules 2004, 37, 256-259.

(22) Chen, F.; Takatsuji, K.; Zhao, D.; Yu, X.; Kumar, S. K.; Tsui, O. $\mathrm{K}$. C. Unexpected thermal annealing effects on the viscosity of polymer nanocomposites. Soft Matter 2017, 13, 5341-5354.

(23) Hu, Y.; Zhou, S.; Wu, L. Surface mechanical properties of transparent poly(methyl methacrylate)/zirconia nanocomposites prepared by in situ bulk polymerization. Polymer 2009, 50, 36093616.

(24) Giovino, M.; Pribyl, J.; Benicewicz, B.; Kumar, S.; Schadler, L. Linear rheology of polymer nanocomposites with polymer-grafted nanoparticles. Polymer 2017, 131, 104-110.

(25) Perez-de-Eulate, N. G.; Sferrazza, M.; Cangialosi, D.; Napolitano, S. Irreversible Adsorption Erases the Free Surface Effect on the $\mathrm{Tg}$ of Supported Films of Poly(4-tert-butylstyrene). ACS Macro Lett. 2017, 6, 354-358. 
(26) Iisaka, K.; Yama, K. S. Mechanical $\alpha$-dispersion and interaction in filled polystyrene and polymethylmethacrylate. J. Appl. Polym. Sci. 1978, 22, 3135-3143.

(27) Bansal, A.; Yang, H.; Li, C.; Cho, K.; Benicewicz, B. C.; Kumar, S. K.; Schadler, L. S. Quantitative equivalence between polymer nanocomposites and thin polymer films. Nat. Mater. 2005, 4, 693698.

(28) Cangialosi, D.; Boucher, V. M.; Alegría, A.; Colmenero, J. Enhanced physical aging of polymer nanocomposites: The key role of the area to volume ratio. Polymer 2012, 53, 1362-1372.

(29) Kontou, E.; Anthoulis, G. The effect of silica nanoparticles on the thermomechanical properties of polystyrene. J. Appl. Polym. Sci. 2007, 105, 1723-1731.

(30) Rittigstein, P.; Torkelson, J. M. Polymer-nanoparticle interfacial interactions in polymer nanocomposites: Confinement effects on glass transition temperature and suppression of physical aging. J. Polym. Sci., Part B: Polym. Phys. 2006, 44, 2935-2943.

(31) Moll, J.; Kumar, S. K. Glass Transitions in Highly Attractive Highly Filled Polymer Nanocomposites. Macromolecules 2012, 45, $1131-1135$.

(32) Hub, C.; Harton, S. E.; Hunt, M. A.; Fink, R.; Ade, H. Influence of sample preparation and processing on observed glass transition temperatures of polymer nanocomposites. J. Polym. Sci., Part B: Polym. Phys. 2007, 45, 2270-2276.

(33) Holt, A. P.; Sangoro, J. R.; Wang, Y.; Agapov, A. L.; Sokolov, A. P. Chain and Segmental Dynamics of Poly(2-vinylpyridine) Nanocomposites. Macromolecules 2013, 46, 4168-4173.

(34) Holt, A. P.; Griffin, P. J.; Bocharova, V.; Agapov, A. L.; Imel, A. E.; Dadmun, M. D.; Sangoro, J. R.; Sokolov, A. P. Dynamics at the Polymer/Nanoparticle Interface in Poly(2-vinylpyridine)/Silica Nanocomposites. Macromolecules 2014, 47, 1837-1843.

(35) Glomann, T.; Schneider, G. J.; Allgaier, J.; Radulescu, A.; Lohstroh, W.; Farago, B.; Richter, D. Microscopic Dynamics of Polyethylene Glycol Chains Interacting with Silica Nanoparticles. Phys. Rev. Lett. 2013, 110, 178001.

(36) Füllbrandt, M.; Purohit, P. J.; Schönhals, A. Combined FTIR and Dielectric Investigation of Poly(vinyl acetate) Adsorbed on Silica Particles. Macromolecules 2013, 46, 4626-4632.

(37) Khatiwada, B. K.; Blum, F. D. Tightly Bound PMMA on Silica Has Reduced Heat Capacities. Langmuir 2019, 35, 11482-11490.

(38) Priestley, R. D.; Rittigstein, P.; Broadbelt, L. J.; Fukao, K.; Torkelson, J. M. Evidence for the molecular-scale origin of the suppression of physical ageing in confined polymer: fluorescence and dielectric spectroscopy studies of polymer-silica nanocomposites. $J$. Phys.: Condens. Matter 2007, 19, 205120.

(39) Burroughs, M. J.; Christie, D.; Gray, L. A. G.; Chowdhury, M.; Priestley, R. D. 21st Century Advances in Fluorescence Techniques to Characterize Glass-Forming Polymers at the Nanoscale. Macromol. Chem. Phys. 2018, 219, 1700368.

(40) Rittigstein, P.; Priestley, R. D.; Broadbelt, L. J.; Torkelson, J. M. Model polymer nanocomposites provide an understanding of confinement effects in real nanocomposites. Nat. Mater. 2007, 6, 278-282.

(41) Priestley, R. D.; Ellison, C. J.; Broadbelt, L. J.; Torkelson, J. M. Structural Relaxation of Polymer Glasses at Surfaces, Interfaces, and In Between. Science 2005, 309, 456-459.

(42) Ellison, C. J.; Kim, S. D.; Hall, D. B.; Torkelson, J. M. Confinement and processing effects on glass transition temperature and physical aging in ultrathin polymer films: Novel fluorescence measurements. Eur. Phys. J. E 2002, 8, 155-166.

(43) Ellison, C. J.; Torkelson, J. M. The distribution of glasstransition temperatures in nanoscopically confined glass formers. Nat. Mater. 2003, 2, 695-700.

(44) Ellison, C. J.; Torkelson, J. M. Sensing the glass transition in thin and ultrathin polymer films via fluorescence probes and labels. J. Polym. Sci., Part B: Polym. Phys. 2002, 40, 2745-2758.

(45) Kim, S.; Roth, C. B.; Torkelson, J. M. Effect of nanoscale confinement on the glass transition temperature of free-standing polymer films: Novel, self-referencing fluorescence method. J. Polym. Sci., Part B: Polym. Phys. 2008, 46, 2754-2764.

(46) Priestley, R. D.; Mundra, M. K.; Barnett, N. J.; Broadbelt, L. J.; Torkelson, J. M.; Priestley, R. D.; Mundra, M. K.; Barnett, N. J.; Broadbelt, L. J.; Torkelson, J. M. Effects of Nanoscale Confinement and Interfaces on the Glass Transition Temperatures of a Series of Poly(n-methacrylate) Films. Aust. J. Chem. 2007, 60, 765-771.

(47) Kim, S.; Torkelson, J. M. Distribution of Glass Transition Temperatures in Free-Standing, Nanoconfined Polystyrene Films: A Test of de Gennes' Sliding Motion Mechanism. Macromolecules 2011, 44, 4546-4553.

(48) Stöber, W.; Fink, A.; Bohn, E. Controlled growth of monodisperse silica spheres in the micron size range. J. Colloid Interface Sci. 1968, 26, 62-69.

(49) Kim, H.; Cang, Y.; Kang, E.; Graczykowski, B.; Secchi, M.; Montagna, M.; Priestley, R. D.; Furst, E. M.; Fytas, G. Direct observation of polymer surface mobility via nanoparticle vibrations. Nat. Commun. 2018, 9, 2918.

(50) Guiselin, O. Irreversible Adsorption of a Concentrated Polymer Solution. Europhys. Lett. 1992, 17, 225-230.

(51) Simavilla, D. N.; Panagopoulou, A.; Napolitano, S. Characterization of Adsorbed Polymer Layers: Preparation, Determination of the Adsorbed Amount and Investigation of the Kinetics of Irreversible Adsorption. Macromol. Chem. Phys. 2018, 219, 1700303.

(52) Jiang, N.; Shang, J.; Di, X.; Endoh, M. K.; Koga, T. Formation Mechanism of High-Density, Flattened Polymer Nanolayers Adsorbed on Planar Solids. Macromolecules 2014, 47, 2682-2689.

(53) Sen, M.; Jiang, N.; Cheung, J.; Endoh, M. K.; Koga, T.; Kawaguchi, D.; Tanaka, K. Flattening Process of Polymer Chains Irreversibly Adsorbed on a Solid. ACS Macro Lett. 2016, 5, 504-508.

(54) Gin, P.; Jiang, N.; Liang, C.; Taniguchi, T.; Akgun, B.; Satija, S. K.; Endoh, M. K.; Koga, T. Revealed Architectures of Adsorbed Polymer Chains at Solid-Polymer Melt Interfaces. Phys. Rev. Lett. 2012, 109, 265501.

(55) Davis, M. J. B.; Zuo, B.; Priestley, R. D. Competing polymersubstrate interactions mitigate random copolymer adsorption. Soft Matter 2018, 14, 7204-7213.

(56) Napolitano, S. Irreversible adsorption of polymer melts and nanoconfinement effects. Soft Matter 2020, 16, 5348-5365.

(57) Jimenez, A. M.; Zhao, D.; Misquitta, K.; Jestin, J.; Kumar, S. K. Exchange Lifetimes of the Bound Polymer Layer on Silica Nanoparticles. ACS Macro Lett. 2019, 8, 166-171.

(58) Kang, E.; Kim, H.; Gray, L. A. G.; Christie, D.; Jonas, U.; Graczykowski, B.; Furst, E. M.; Priestley, R. D.; Fytas, G. Ultrathin Shell Layers Dramatically Influence Polymer Nanoparticle Surface Mobility. Macromolecules 2018, 51, 8522-8529.

(59) Kang, E.; Graczykowski, B.; Jonas, U.; Christie, D.; Gray, L. A. G.; Cangialosi, D.; Priestley, R. D.; Fytas, G. Shell Architecture Strongly Influences the Glass Transition, Surface Mobility, and Elasticity of Polymer Core-Shell Nanoparticles. Macromolecules 2019, $52,5399-5406$.

(60) Fetters, L. J.; Hadjichristidis, N.; Lindner, J. S.; Mays, J. W. Molecular Weight Dependence of Hydrodynamic and Thermodynamic Properties for Well-Defined Linear Polymers in Solution. J. Phys. Chem. Ref. Data 1994, 23, 619-640.

(61) Fujii, Y.; Yang, Z.; Leach, J.; Atarashi, H.; Tanaka, K.; Tsui, O. K. C. Affinity of Polystyrene Films to Hydrogen-Passivated Silicon and Its Relevance to the $\mathrm{Tg}$ of the Films. Macromolecules 2009, 42, $7418-7422$

(62) Ellison, C. J.; Mundra, M. K.; Torkelson, J. M. Impacts of Polystyrene Molecular Weight and Modification to the Repeat Unit Structure on the Glass Transition-Nanoconfinement Effect and the Cooperativity Length Scale. Macromolecules 2005, 38, 1767-1778.

(63) Ellison, C. J.; Ruszkowski, R. L.; Fredin, N. J.; Torkelson, J. M. Dramatic Reduction of the Effect of Nanoconfinement on the Glass Transition of Polymer Films via Addition of Small-Molecule Diluent. Phys. Rev. Lett. 2004, 92, 095702.

(64) Hao, Z.; Ghanekarade, A.; Zhu, N.; Randazzo, K.; Kawaguchi, D.; Tanaka, K.; Wang, X.; Simmons, D. S.; Priestley, R. D.; Zuo, B. 
Mobility Gradients Yield Rubbery Surfaces on Top of Polymer Glasses. Nature 2021, 596, 372-376.

(65) Zha, H.; Wang, Q.; Wang, X.; Cangialosi, D.; Zuo, B. Enhanced Free Surface Mobility Facilitates the Release of Free-Volume Holes in Thin-Film Polymer Glasses. Macromolecules 2021, 54, 2022-2028.

(66) Napolitano, S.; Cangialosi, D. Interfacial Free Volume and Vitrification: Reduction in Tg in Proximity of an Adsorbing Interface Explained by the Free Volume Holes Diffusion Model. Macromolecules 2013, 46, 8051-8053.

(67) Zhang, C.; Guo, Y.; Priestley, R. D. Glass Transition Temperature of Polymer Nanoparticles under Soft and Hard Confinement. Macromolecules 2011, 44, 4001-4006.

(68) Sharp, J. S.; Forrest, J. A. Free Surfaces Cause Reductions in the Glass Transition Temperature of Thin Polystyrene Films. Phys. Rev. Lett. 2003, 91, 235701.

(69) Starr, F. W.; Douglas, J. F.; Meng, D.; Kumar, S. K. Bound Layers "Cloak" Nanoparticles in Strongly Interacting Polymer Nanocomposites. ACS Nano 2016, 10, 10960-10965. 